\title{
A two-phase solid-fluid model for dense granular flows including dilatancy effects: comparison with submarine granular collapse experiments
}

\author{
F. Bouchut ${ }^{1}$, E.D. Fernández-Nieto ${ }^{2}$, E.H. Koné ${ }^{3}$, A. Mangeney ${ }^{3, \star}$, and G. Narbona-Reina ${ }^{2}$ \\ ${ }^{1}$ Université Paris-Est, Laboratoire d'Analyse et de Mathématiques Appliquées (UMR 8050), CNRS, UPEM, UPEC, F-77454, \\ Marne-la-Vallée, France \\ ${ }^{2}$ Universidad de Sevilla, Dpto. Matemática Aplicada I \& IMUS, Universidad de Sevilla. E.T.S. Arquitectura, 41012 Sevilla, Spain \\ ${ }^{3}$ Université Paris Diderot, Sorbone Paris Cité, Institut de Physique du Globe de Paris, 75005 Paris, France
}

\begin{abstract}
We simulate here the collapse of granular columns immersed in a viscous fluid based on a simplified version of the model developed by [2]. The simulation quite well reproduces the dynamics and deposit of the granular mass as well as the excess pore fluid pressure measured in the laboratory experiments of [10] owing that dilatancy effects and pore pressure feedback are accounted for. In particular, the difference in the behaviour of initially loose and dense columns is reproduced numerically.
\end{abstract}

\section{Introduction}

Describing grain/fluid interaction in debris flows models is still an open and challenging issue with important impact on hazard assessment $[3,7]$. A key issue in modeling grain/fluid flows is to describe the compression/dilation of the granular phase and its interaction with the pore fluid pressure. This is particularly challenging when trying to include these effects in models which are classically used for real applications, that assume thin-layer flows [1, 2, 9].

The depth-averaged thin-layer model used here is essentially an extension to non-uniform flows of the model proposed by [9]. We consider a two-phase layer that models the grain/fluid mixture with a fluid layer on top of it to account for fluid transfer into and/or out of the mixture [2]. The model describes explicitly the velocity of the solid and fluid phases, the compression/dilation of the granular media and its interaction with the pore fluid pressure. The system of mass and momentum conservation equations from Jackson's model is closed by a weak compressibility relation following [11]. This relation implies that the occurrence of dilation or contraction of the granular material in the model depends on whether the solid volume fraction is respectively higher or lower than a critical value. This description of dilatancy has been used in [9] to develop a thin-layer depth-averaged two-phase model for immersed granular flows (see also [6]). Moreover, following [9], the critical value is assumed to depend on the shear rate and the solid pressure.

Two models have been proposed in [2], depending on the order of magnitude of the friction coefficient between the two phases within the mixture. In both cases, the model reproduces the expected behaviour (see e. g. [9]): when dilation occurs, the fluid is sucked into the granular

\footnotetext{
^e-mail: mangeney@ipgp.jussieu.fr
}

material, the pore pressure decreases and the friction force on the granular phase increases. On the contrary, in the case of contraction, the fluid is expelled from the mixture, the pore pressure increases and the friction force diminishes. The extra upper fluid layer considered in the model is essential to be consistent with the compression/dilation effects since it allows the fluid to be expelled or sucked from/in the mixture at its upper boundary. Additionally, this configuration also allows to use the model for the case of immersed flows, which corresponds to the experiments investigated in [10] and simulated here.

In this paper we present the first simulations obtained by solving numerically the equations proposed in [2] and compare the results to the laboratory experiments of granular column collapse immersed in a viscous fluid performed by [10]. These experiments have been chosen because they clearly show and quantify the strong impact of the initial compaction on the flow dynamics and deposit of the granular mass. Indeed, the initially loose column travels much further than the initially dense column due to the dilatancy processes described above. These simulations show that the model is able to capture at a first order the main effect of compression/dilation and its coupling with the pore fluid pressure.

In Section 2 the model and parameter settings are described and in Section 3 a quantitative comparison between simulation and experiments is presented and discussed.

\section{Grain/fluid Model with dilatancy}

\subsection{Equations}

The two-layer model proposed in [2] is made of a fluid layer on top of a two-phase mixture layer (Figure 1). It is described by three mass and three momemtum conservation equations and a closure relation. Moreover, it includes 
mass transference between the upper fluid layer and the fluid phase within the mixture layer. Let us denote by $h_{f}$ the thickness of the upper only-fluid layer and $\mathbf{u}_{f}$ its velocity, by $h_{m}$ the thickness of the mixture layer, $\varphi$ the solid volume fraction, $\mathbf{v}$ the solid phase velocity, and $\mathbf{u}$ the fluid phase velocity in the mixture layer. Then, the three continuity equations defining the model are:

$$
\begin{gathered}
\partial_{t}\left(\varphi h_{m}\right)+\nabla \cdot\left(\varphi h_{m} \mathbf{v}\right)=0, \\
\partial_{t}\left((1-\varphi) h_{m}\right)+\nabla \cdot\left((1-\varphi) h_{m} \mathbf{u}\right)=-\mathcal{V}_{f}, \\
\partial_{t} h_{f}+\nabla \cdot\left(h_{f} \mathbf{u}_{f}\right)=\mathcal{V}_{f},
\end{gathered}
$$

with $\mathcal{V}_{f}$ the fluid mass transference. The evolution of solid volume fraction is described by

$$
\partial_{t} \varphi+\mathbf{v} \cdot \nabla \varphi=-\varphi \Phi
$$

where $\Phi$ is the closure function that takes into account dilatancy effects. From (1), (2), (3) and (4), one can indeed deduce the value of the fluid mass transference

$$
\mathcal{V}_{f}=-h_{m} \Phi-\nabla \cdot\left((1-\varphi) h_{m}(\mathbf{u}-\mathbf{v})\right)
$$

Following the closure proposed by Roux and Radjai [11] we set $\Phi=K \dot{\gamma}\left(\varphi-\varphi_{c}^{e q}\right)$, where the critical-state compacity is $\varphi_{c}^{e q}=\varphi_{c}^{\text {stat }}-K_{2} \frac{\eta_{f} \dot{\gamma}}{p_{s \mid b}}$ (see [9]). $K$ and $K_{2}$ are two constants, $\varphi_{c}^{\text {stat }}$ is a constant volume fraction corresponding to the static equilibrium, $\eta_{f}$ is the fluid viscosity, $\dot{\gamma}$ the strain rate and $p_{s \mid b}$, the solid pressure at the base of the flow (see $[2,9]$ for more details).

The momentum equations involve the bottom solid pressure $p_{s \mid b}=\left(\left(\rho_{s}-\rho_{f}\right) g_{c} \varphi h_{m}-\left(p_{f m}^{e}\right)_{b}\right)_{+}$through the effective bottom solid friction

$$
\widetilde{\tau}_{b}=p_{s \mid b} \tan \delta_{\text {eff }}+K_{1} \eta_{f} \dot{\gamma}
$$

where $\tan \delta_{\text {eff }}=\tan \delta+K\left(\varphi-\varphi_{c}^{e q}\right), \rho_{f}$ and $\rho_{s}$ are the density of the fluid and the solid, respectively, $g_{c}=g \cos \theta$, with $g$ the gravity acceleration constant and $\theta$, the slope of the inclined plane. $K_{1}$ is a constant and the excess pore pressure $p_{f_{m}}^{e}$ is such that the total fluid and solid pressures are

$$
\begin{aligned}
& p_{f}(z)=\rho_{f} g_{c}\left(b+h_{m}+h_{f}-z\right)+p_{f_{m}}^{e}(z), \\
& p_{s}(z)=\left(\rho_{s}-\rho_{f}\right) g_{c} \varphi\left(b+h_{m}-z\right)-p_{f_{m}}^{e}(z)
\end{aligned}
$$

where $b$ denotes the bottom elevation with respect to the inclined plane, and where $p_{f_{m}}^{e}$ is the average with respect to $z$ of $p_{f_{m}}^{e}(z)$. The main difference between a hydrostatic model and the one derived here is the contribution of $p_{f_{m}}^{e}$ in the solid friction (6) and its averaged gradient, that is defined as follows:

$$
\overline{\nabla p_{f_{m}}^{e}}=\frac{1}{h_{m}}\left(\nabla\left(h_{m} p_{f_{m}}^{e}\right)+\left(p_{f_{m}}^{e}\right)_{b} \nabla b\right) .
$$

Two definitions are obtained in [2] for $\left(p_{f_{m}}^{e}\right)_{\mid b}$ and $p_{f_{m}}^{e}$. In this work we consider the simplest case, associated to a large friction $\beta=150 \eta_{f} \varphi^{2} / d^{2}(1-\varphi)=O\left(\epsilon^{-1}\right)$ between the phases. $\epsilon$ is the aspect ratio assumed to be small according to the thin-layer framework and $d$ is the solid particles mean diameter (see [2] for details). Hence

$$
\left(p_{f_{m}}^{e}\right)_{\mid b}=-\frac{\beta}{(1-\varphi)^{2}} \frac{h_{m}^{2}}{2} \Phi, \quad p_{f_{m}}^{e}=-\frac{\beta}{(1-\varphi)^{2}} \frac{h_{m}^{2}}{3} \Phi .
$$

To simulate underwater granular flows, we consider the following simplifications: (i) we rewrite the hydrostatic pressure terms in the momentum equations by using the hypothesis of horizontal free surface: $h_{m}(t, x)+$ $h_{f}(t, x)+b(x)+x \tan \theta=c s t$ (Figure 1) and (ii) we neglect the friction between the upper fluid layer and the mixture layer. Moreover, we consider that the only-fluid layer has no tangential movement (i. e. $\mathbf{u}_{f} \equiv 0$ ). Let the fluid and solid contributions in the mixture mass be denoted by $h_{1}=(1-\varphi) h_{m}$ and $h_{2}=\varphi h_{m}$ and the fluid and solid discharges by $\mathbf{q}_{1}=h_{1} \mathbf{u}$ and $\mathbf{q}_{2}=h_{2} \mathbf{v}$, respectively. The system summarizes then in conservative form as

$$
\left\{\begin{aligned}
\partial_{t} h_{1}+\nabla \cdot \mathbf{q}_{1}=-\mathcal{V}_{f}, & \\
\partial_{t} \mathbf{q}_{1}+\nabla \cdot\left(\mathbf{u} \otimes \mathbf{q}_{1}\right) & =-\frac{1}{2} \mathcal{V}_{f} \mathbf{u}-\mathbf{f}, \\
\partial_{t} h_{2}+\nabla \cdot \mathbf{q}_{2}=0, & \\
\partial_{t} \mathbf{q}_{2}+\nabla \cdot\left(\mathbf{v} \otimes \mathbf{q}_{2}\right) & =-\frac{1-r}{2} g_{c} \nabla\left(\varphi h_{m}^{2}\right)+r \mathbf{f} \\
& -(1-r) g_{c} h_{2} \nabla \hat{b}-\frac{\widetilde{\tau}_{b}}{\rho_{s}} \operatorname{sgn}(\mathbf{v}),
\end{aligned}\right.
$$

where $r=\frac{\rho_{f}}{\rho_{s}}, \hat{b}=b+x \tan \theta, \mathcal{V}_{f}$ is given by (5) and

$$
\mathbf{f}=\frac{1}{\rho_{f}}\left(h_{1} \overline{\nabla p_{f m}^{e}}+\beta h_{m}(\mathbf{u}-\mathbf{v})\right)
$$

\subsection{Numerical method}

The model is discretized with a combination of finite volume and finite difference schemes. The finite volume scheme is a Roe-type non-conservative method that handles the advection-convection part of the system, while a standard finite difference scheme deals with the source terms of orders 0 and 2 .

As we will simulate the spreading of a granular column immersed in a fluid (see section 3.1), the model should be able to deal with the transition between the cases $h_{m} \neq 0$ and $h_{m}=0$ (so-called dry bottom surface in numerical methods). This is a key and generally difficult issue in the design of numerical schemes solving hyperbolic equations. It is noteworthy that the current stage of the solver blows up when we use dry bottom surface to simulate granular column collapse. This is likely a numerical issue that we are investigating now. As a first step to simulate the experiments, we added here a thin artificial layer of 1 $\mathrm{mm}$ to the bottom surface. This is enough to prevent the code to blow up even though we may notice very small oscillations on the simulated profiles on the top of the mass for the dense initial packing and ahead of the front for the two packing configurations (Figure 2).

\section{Simulation of granular collapses}

\subsection{Laboratory experiments}

We simulate here the collapse of a granular column initially released from rest in a viscous liquid that has been 


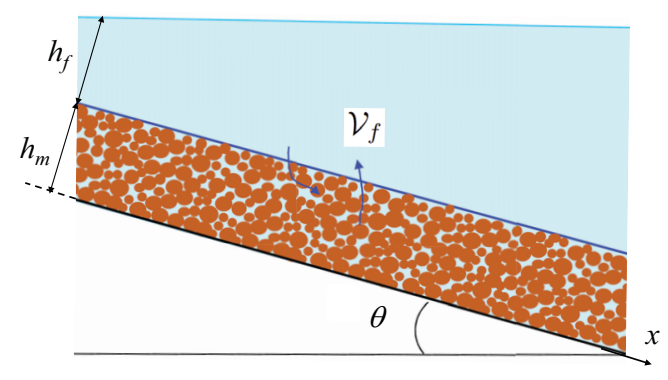

Figure 1. Immersed configuration.

experimentally investigated by [10]. The experimental setup consists of a box $70 \mathrm{~cm}$ long, $15 \mathrm{~cm}$ wide and $15 \mathrm{~cm}$ high, full of a viscous liquid. The horizontal bottom of the box is made rough by gluing a layer of particles on it. This experiment corresponds to a one-dimensional dambreak with an initial rectangular mixture mass of height $H_{i}$ and width $L_{i}$ that is at rest with a volume fraction $\varphi_{i}$. The release of an initially loose and initially dense granular column is tested experimentally with the data $\left(\varphi_{i}=0.55, L_{i}=6 \mathrm{~cm}, H_{i}=4.8 \mathrm{~cm}\right)$ and $\left(\varphi_{i}=0.6, L_{i}=6\right.$ $\left.\mathrm{cm}, H_{i}=4.2 \mathrm{~cm}\right)$, respectively. In both cases, the particles are glass beads of density $\rho_{s}=2500 \mathrm{~kg} \cdot \mathrm{m}^{-3}$ and mean diameter $d=225 \mu \mathrm{m}$, whereas the fluid has a density $\rho_{f}=1000 \mathrm{~kg} \cdot \mathrm{m}^{-3}$ and a viscosity $\eta_{f}=12 \times 10^{-3}$ Pa.s. For these experiments, we have $\varphi_{c}^{\text {stat }}=0.585, \tan \delta=0.32$ and we set $K=4.09, K_{1}=90.5$ and $K_{2}=25$ in the model as in [9].

\subsection{Simulations}

Overall, the quantitative agreement between simulation and observation is quite good (Figures 2,3). The key point is that the spreading of the granular mass is, as stated in [10], mainly controlled by its initial volume fraction, with two different regimes corresponding to dense and loose packings. Loose packing columns have fast dynamics and lead to thin and long deposits, while dense ones produce slow flows and short runout distances (Figures 2 and 3(a)). Furthermore the maximum thickness of the deposit is much smaller for the initially loose case. While the deposit is quite well reproduced, the simulated mass spreads more rapidly than in the experiments. This is a common feature obtained when comparing thin-layer depth-averaged models with granular collapse experiments [4, 8]. Furthemore the effect of the gate that releases the granular column is not taken into account in the model. It has however a nonnegligible effect on the flow dynamics while it almost does not change the deposit (see Figures 14 and 15 of [5]).

In addition, a positive (resp. negative) pore pressure is simulated below the column for loose (resp. dense) packings as in the experiments (Figure 3(b)). In case of dilation, the fluid is sucked into the mixture decreasing the pore fluid pressure and increasing thereby the effective friction on the granular phase. On the other hand, contraction induces an expulsion of the fluid from the mix- ture increasing the pore fluid pressure and decreasing the effective friction. This so-called pore pressure feedback claimed in [10] and references therein is well reproduced in the simulation. In the experiments, pressures are measured at the position $x=2 \mathrm{~cm}$ from the wall $(x=0)$. We represent here the simulated pressure at $x=2 \mathrm{~cm}$ (black), $x=3 \mathrm{~cm}$ (blue), $x=4 \mathrm{~cm}$ (red).

For the loose case, the simulated value of the pore pressure at $x=2 \mathrm{~cm}$ and its time change is in quite good agreement with the pressure measured at $x=2 \mathrm{~cm}$. The model simulates a higher pick fluid pressure at the very beginning of the collapse which is not measured experimentally, at least at the acquisition rate of these measurements. For the dense case, even though the simulated pore pressure is of the good order of magnitude, it is quite different from the measured pressure. In particular, the pressure decrease at $x=2 \mathrm{~cm}$, starts much later than in the experiments. When going closer to the initial gate position (i. e. $x=3,4 \mathrm{~cm}$ ), the pressure change starts earlier, as observed at $x=2$ $\mathrm{cm}$ in the experiments. This time shift is only seen in the dense case and may correspond to the strong effect of the gate in that case or to more fundamental modeling issue.

Let us now compare the results obtained with the nonhydrostatic pressure (i. e. the whole model involving the excess pore fluid pressure and its gradient) with those obtained when only hydrostatic pressure is assumed (i. e. with $\left.p_{f m}^{e}=0\right)$. Figure 2 clearly shows that the whole model results (middle plots) are much closer to the experiments (top plots) than the hydrostatic model (bottom plots), in particular in the loose case. The same observations holds for Figure 3(a), where we plot the time evolution of the front position. These results point out the relevance of including the excess pore fluid pressure. However, the numerical discretization of the excess pore fluid pressure and of its gradient is a very challenging process that is still under investigation. Improving the discretization may resolve the inability of the solver to simulate the granular collapse over a dry bottom surface and/or reduce the observed discrepancy in the triggering time of the pressure decrease.

\section{Conclusion}

The two-layer two-phase solid/fluid model introduced in [2] is solved here numerically in the case of immersed granular flows. This thin-layer depth-averaged model makes it possible for the fluid to be either expelled from or sucked into the mixture at its upper boundary depending on the compression or dilation of the granular phase. Simulations with the current stage of the solver are compared to the experiment of immersed granular collapse performed by [10]. We show that the quantitative behaviour is quite well reproduced by the model in terms of front dynamics, deposit and pore fluid pressure change with time. In particular, the simulation reproduces the strong change in behaviour of initially dense and loose columns. Discrepancies between the simulation and experiments are however observed. They are due to the depth-averaged thin-layer approximation, to the absence of the gate in the simulation but also probably to numerical issues. Indeed, the discretization of the pressure and 

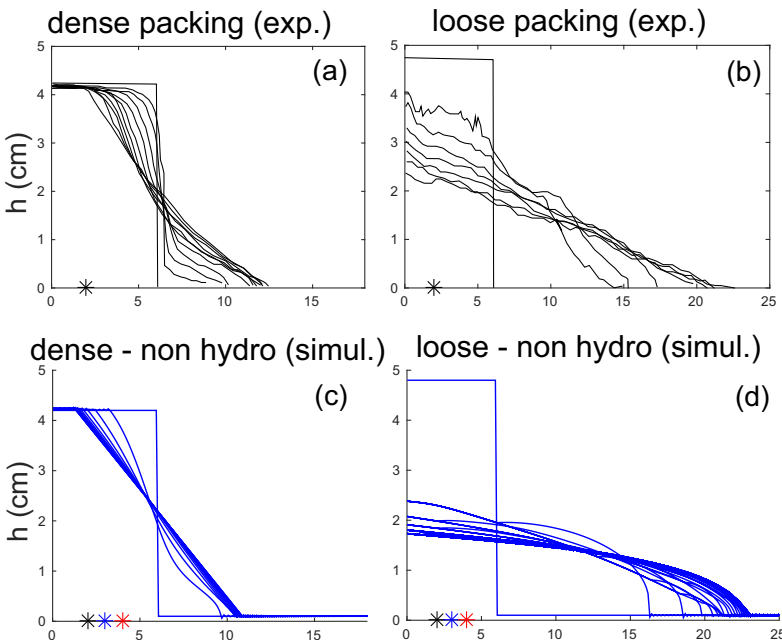

(c)
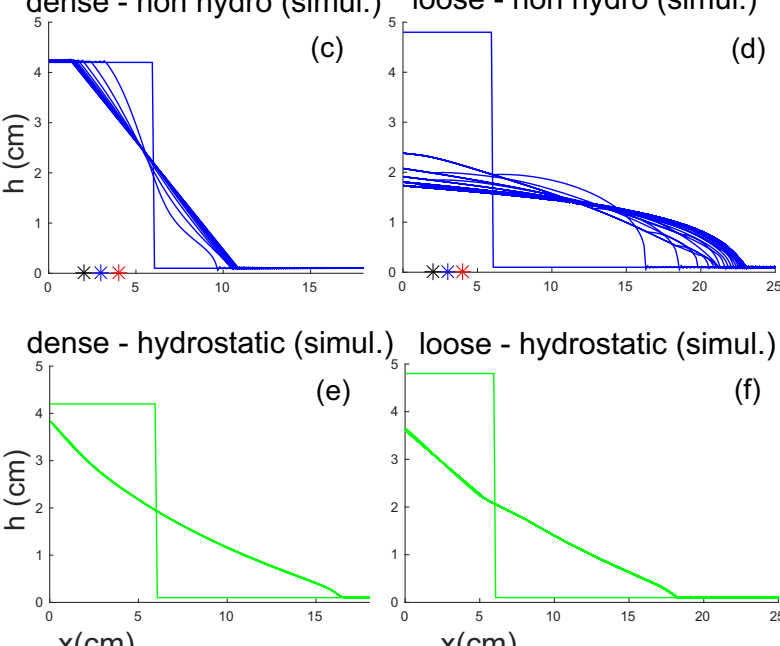

(e)

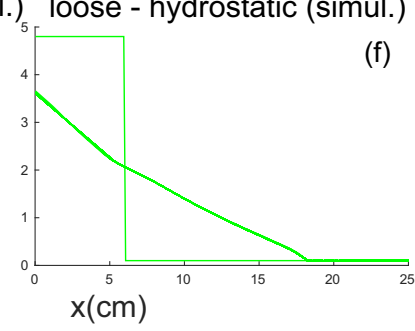

Figure 2. Granular mass profiles from experiments [top] and simulations (non hydrostatic [middle] and hydrostatic [bottom] pressures). Left: dense initial packing $\left(\varphi_{i}=0.6, L_{i}=6 \mathrm{~cm}, H_{i}=\right.$ $4.2 \mathrm{~cm})$ every $3 \mathrm{~s}$. Right: loose initial packing $\left(\varphi_{i}=0.55, L_{i}=6\right.$ $\left.\mathrm{cm}, H_{i}=4.8 \mathrm{~cm}\right)$ every $0.66 \mathrm{~s}$. The marker $*$ is the position where the time series of the excess pore fluid pressure is measured in experiments.

of its gradients as well as the numerical treatment of the front should be improved to go further in the quantitative comparison with the experiments. This is under investigation. We also show that when cancelling the excess pore fluid pressure by assuming hydrostatic pressure, very strong difference is observed with the experiments in terms of dynamics and deposit of the granular mass.

\section{Acknowledgements}

This work has been funded by the ANR-11-BS010016 LANDQUAKES, the ERC-CG-2013-PE10-617472 SLIDEQUAKES and FEDER and the Spanish Government through the research project MTM2015-70490-C22-R.

\section{References}

[1] Bouchut, F., Fernandez-Nieto, E. D., Mangeney, A., and Narbona-Reina, G., A two-phase shallow debris flow model with energy balance, Math. Model. Num. Anal., 49(1), 101-140 (2015).

[2] F. Bouchut, E.D. Fernández-Nieto, A. Mangeney, G. Narbona-Reina, A two-phase two-layer model for fluidized granular flows with dilatancy effects. J. Fluid Mech, 801, 166-221 (2016).
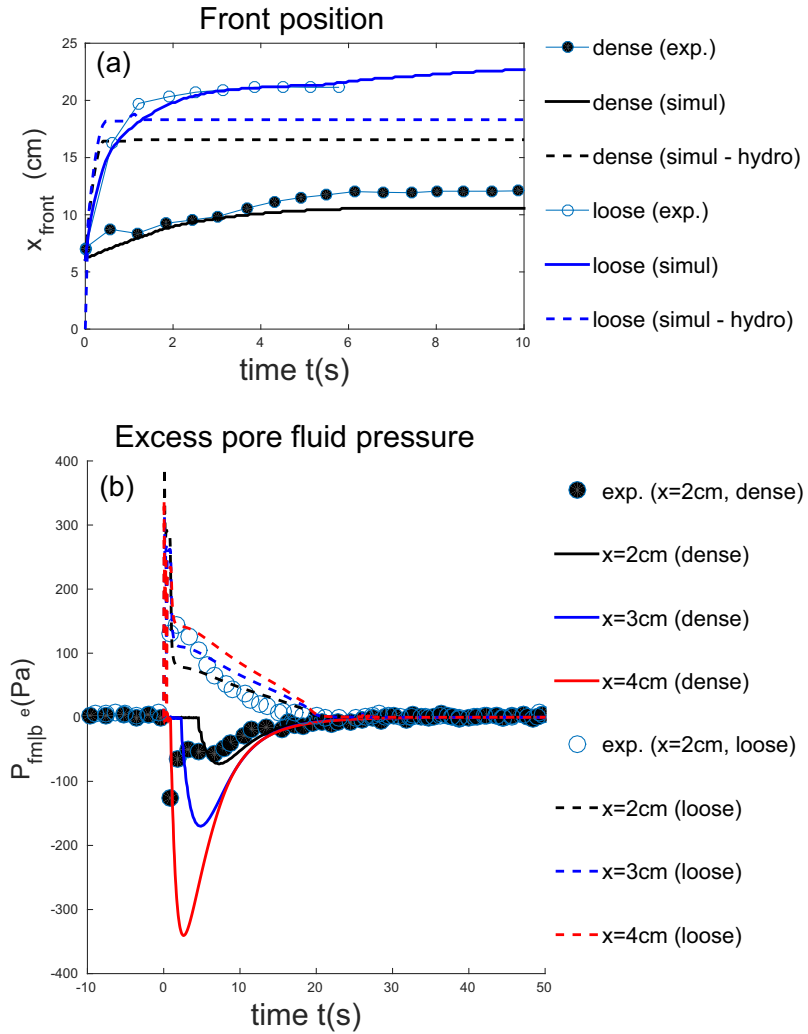

Figure 3. Time evolution of (a) the front position for both initial packing from experiments (dense - - and loose -o-) and simulations (non hydrostatic [dense -, loose -] and hydrostatic [dense --, loose --] pressures) and (b) the pore pressure below the column from experiments (dense $\bullet$ and loose $\circ$ ) at $x=2 \mathrm{~cm}$ and simulations at $x=2 \mathrm{~cm}$ (black), $x=3 \mathrm{~cm}$ (blue), $x=4 \mathrm{~cm}$ (red).

[3] Delannay, R., Valance, A., Mangeney, A., Roche, O., and Richard, P., Granular and particle-laden flows: from laboratory experiments to field observations, J. Phys. D: Appl. Phys., in press (2016).

[4] Fernandez-Nieto, E. D., Garres-Diaz, J., Mangeney, A., and Narbona-Reina, G., A multilayer shallow model for dry granular flows with the mu(I) rheology: Application to granular collapse on erodible beds, J. Fluid. Mech., 798, 643-681 (2016).

[5] Ionescu, I., Mangeney, A., Bouchut, F., and Roche, O., Viscoplastic modeling of granular column collapse with pressure-dependent rheology, J. Non-Newtonian Fluid. Mech., 219, 1-18 (2015).

[6] R. M. Iverson and D. L. George, A depth-averaged debris-flow model that includes the effects of evolving dilatancy. I. Physical basis. Proc. R. Soc. A, 470:0 20130819 (2014)

[7] R. M. Iverson, M. Logan, R. G. LaHusen, and M. Berti, The perfect debris flow? Aggregated results from 28 large-scale experiments. J. Geophys. Res., 115:0 F03005 (2010).

[8] Mangeney-Castelnau, A., Bouchut, F., Vilotte, J.P., Lajeunesse, E., Aubertin, A., and Pirulli, M., On the use of Saint Venant equations to simulate the spreading of a granular mass, J. Geophys. Res., 110, B09103 (2005). 
[9] M. Pailha and O. Pouliquen, A two-phase flow description of the initiation of underwater granular avalanches. J. Fluid Mech., 633:0, 115-135 (2009).

[10] L. Rondon, O. Pouliquen and P. Aussillous, Granular collapse in a fluid: Role of the initial volume frac- tion, Physics of Fluids, 23, 073301 (2011)

[11] S. Roux and F. Radjai, Texture-dependent rigid plastic behaviour. In H. J. Herrmann et al., editor, Physics of Dry Granular Media, 305-311. Kluwer, (1998). 